\title{
The LickX spectra ${ }^{\star}$ (Research Note)
}

\author{
G. Worthey ${ }^{1}$, A. B. Danilet ${ }^{1}$, and S. M. Faber ${ }^{2}$ \\ 1 Department of Physics and Astronomy, Washington State University, 1245 Webster Hall, Pullman WA 99164, USA \\ e-mail: gworthey@wsu.edu \\ 2 UCO/Lick Observatories, 1156 High Street, Santa Cruz CA 95064, USA
}

Received 15 July 2013 / Accepted 19 November 2013

\begin{abstract}
Context. Collections of stellar spectra, often called stellar libraries, are useful in a variety of applications in the field of stellar populations.

Aims. This is an attempt to improve the much-used Lick library of stellar spectra by removing jitter from the wavelength scale via cross-correlation, and calling the result the LickX library.

Methods. Each spectrum was cross-correlated with a template spectrum and a new wavelength solution sought. Low-order polynomials were fit to adjust the old scale to a new fit. Indices were measured, new standard star averages found, and adjusted averages derived for the program stars.

Results. The greatest gains in accuracy are expected for the fainter stars and stars of extreme surface temperatures; the bright K giant standard stars in LickX have the same uncertainties as Lick. The spectra and a table of index measurements in which repeated measurements are averaged are made available electronically.
\end{abstract}

Key words. catalogs - stars: abundances - galaxies: stellar content - stars: fundamental parameters

\section{Introduction}

A historically influential set of spectra based upon nonspectrophotometric observations carried out at Lick Observatory between 1972 and 1984 using the red-sensitive image dissector scanner (IDS; Robinson \& Wampler 1972) and Cassegrain spectrograph at Lick Observatory. The spectra ranged from about 4000 to $6400 \AA$ with spectral resolutions between 8 and $10 \AA$. The spectra were not fluxed, not even relatively, but were divided by a quartz-iodide tungsten lamp spectrum.

Stellar spectral libraries that, like the Lick library, span different metallicities as well as temperatures and luminosities, e.g., MILES (Sánchez-Blázquez et al. 2006; Falcón-Barroso et al. 2011), NGSL (Heap \& Lindler 2010), and Indo-US (Valdes et al. 2004), are crucially important for a variety of applications in stellar populations. And, while it may seem that the Lick library might be outmoded, it is still useful in at least two regards. First, the accuracies of some indices with long wavelength spans (e.g., $\left.\mathrm{Mg}_{1}, \mathrm{Mg}_{2}\right)$ continue to be competitive with and in most cases exceed measurements take from CCD spectra. The exact reasons why remain obscure. Secondly, the target selection contains stars in odd but sometimes crucial corners of parameter space that are not well represented in other libraries.

A set of 11 pseudo equivalent width indices were measured by hand for stars and galaxies early on (Burstein et al. 1984; Faber et al. 1985; Burstein et al. 1986; Gorgas et al. 1993) and then expanded to a list of 25 indices (Worthey et al. 1994; Worthey \& Ottaviani 1997). The latter papers also introduced an

* Individual stellar spectra, in FITS files, and the ascii catalog of absorption feature index strengths are only available at the CDS via anonymous ftp to cdsarc.u-strasbg. fr (130.79.128.5) or via http://cdsarc.u-strasbg.fr/viz-bin/qcat?]/A+A/561/A36 automated way to measure the indices wherein cross-correlation in the neighborhood of each index was used to center the index in wavelength, and where one particular observation of one giant, HR 6018, was used as the wavelength fiducial. Transferral of this wavelength scale to hot and cool stars was done stepwise through pairs of other template spectra.

\section{Data available}

In this paper, we reprocess the stellar spectra of the Lick library. The results are, first, increased accuracy in which the considerable uncertainties of wavelength scale are much reduced, and, second, a collection of spectra that can be used with fair ease by the community, since the improved wavelength scales are placed on a simple linear scale, albeit at the twilight of the usefulness of this set of spectra.

Methodologically, the spectra were subdivided into (typically) 15 pieces. Each was cross-correlated with a rebinned, high resolution spectrum, mostly synthetically generated from Buser \& Kurucz (1992) machinery, but a cool M giant from the Elodie library (Prugneil \& Soubiran 2001) was used to center the coolest stars. The wavelength scales of the templates are good to small fractions of an Angstrom. The resulting shifts were displayed as a function of wavelength, wild points rejected, and fit with a polynomial. The polynomial most often used was a simple line, and the second most common case was an even more simple shift in the starting wavelength. This indicates that local shifts in the spectra were not as bad as had been assumed in the 1980s and 1990s, and that the automated method used then probably introduced noise in the wavelength placement for each index. On the other hand, many spectra did require quadratic or 
cubic solutions. If the solution was nonlinear, the spectrum was rebinned to be on a linear wavelength scale at the end.

During the process, beginning and ending wavelengths were also noted, and these were from time to time, more conservative than the original, so a few index measurements are trimmed in the LickX library compared to the original. As an indication of the jitter removed, the original wavelength scales and the crosscorrelated versions we produce agree within a dispersion of $\sigma=$ $3.3 \AA$, with approximate average accord in the blue, but with the new wavelength scales trending to about $0.5 \AA$ redder at the red end of the spectrum.

The data are presented in FITS format files. Keywords wcrval and wcdelt give the wavelength of the first pixel in Angstroms, and the difference in wavelength between any two pixels in Angstroms per pixel, respectively. Occasionally, keywords wlimit1 and wlimit 2 were inserted. These give the blue and red wavelengths, respectively, that delimit the bounds of what we consider the useful portion of each spectrum. They are often more restrictive than those generated by the automated algorithm (the algorithm finds a spectrum endpoint by testing for the presence of 10 consecutive pixels greater than $1 / 2000$ the number of counts in the maximum pixel).

When the revisions of the spectra were complete, some spectral indices were measured. The indices are the 25 from Worthey et al. (1994), Worthey \& Ottaviani (1997), with wavelength definition updates from Trager et al. (1998), some of the 79 indices given by Serven et al. (2005) that fall into the spectral range of the Lick spectra, three emission line indices from González (1993), and the modified $\mathrm{H} \beta_{0}$ index of Cervantes \& Vazdekis (2009) for a total of 63 spectral indices. The definitions are summarized in Table 1. The spectra were smoothed a small amount in the middle of the spectral range, so that they nowhere fell below an instrumental resolution equivalent to a velocity dispersion of $200 \mathrm{~km} \mathrm{~s}^{-1}$ for reasons of eventually producing a consistent set of spectra indices at this resolution. The smoothing had negligible effect on the indices.

There are 455 unique stars amongst the 1043 spectra, for the most part including a set of line strength standard stars observed in each of the 61 observing runs except for run 53 (HR 489, HR 1805, HR 2002, HR 2600, HR 3905, HR 6018, HR 6770, HR 7429, HR 7576). Data were not obtained in some runs. As in Worthey et al. (1994), these line strength standards define the system and then were used to generate additive corrections on a run by run basis if the standard stars in any particular run and in any particular index were statistically different from the grand average. A difference from Worthey et al. (1994) is that the averaging of multiply-observed stars was done using the Tukey biweight (Tukey 1958) as estimator for location and scale, as opposed to outlier-culled means and standard deviations. The biweight scales performed on the wavelength-rectified spectra were systematically smaller than the previous standard deviations measured from the unrectified spectra, and this tended to increase the number of run corrections. Another detail is that runs 8 and 53 had insufficient standard star observations for good statistics, and so the whole list of stars (less those two runs) were used as secondary standards to correct the indices from those two runs. The single-measurement uncertainties listed in the Table 1 are biweight scales, which reduce to standard deviations in the case of Gaussian random statistics.

Figure 1 gives a sample of the agreement between the old automated measurement and the results of simply measuring the newly cross-correlated specra using the new wavelength scales, for two spectral indices with broad dynamic range, $\mathrm{H} \beta$ and $\mathrm{NaD}$,
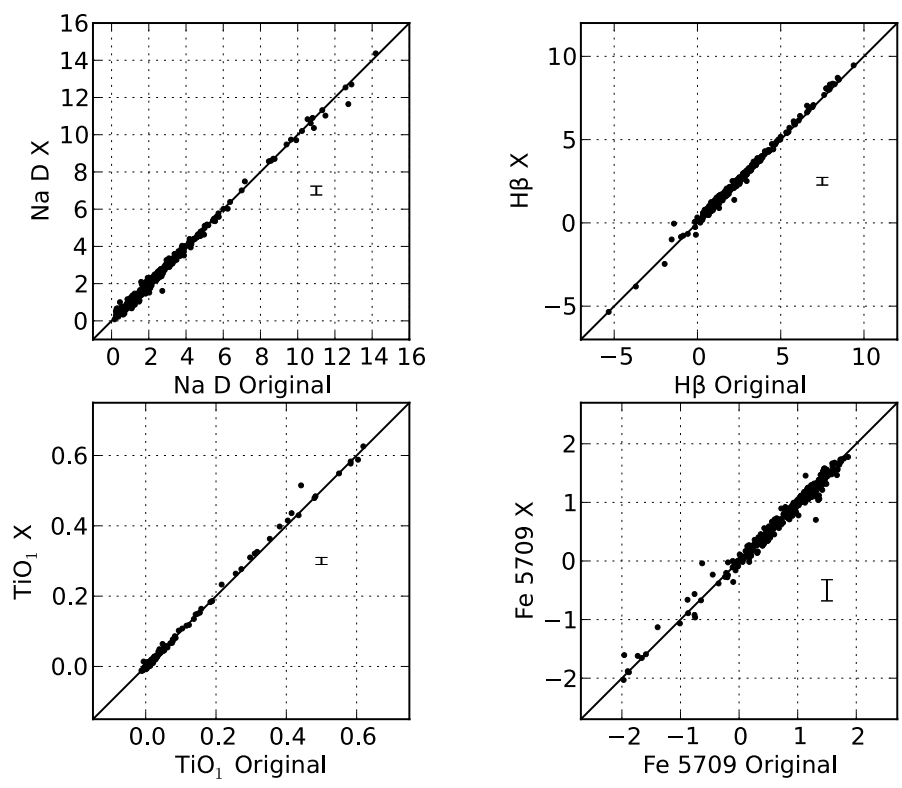

Fig. 1. Example indices $\left(\mathrm{H} \beta, \mathrm{Na} \mathrm{D}, \mathrm{TiO}_{1}\right.$, and $\left.\mathrm{Fe} 5709\right)$ averaged from the original Lick library and from the new wavelength rectified version. Single-measurement error bars are shown for comparison. Apparently, the errors induced by wavelength scale changes are almost random, with no systematic drifts, with amplitudes comparable to, but somewhat smaller than, the single-measurement error.

an index that should be insensitive to wavelenth errors, $\mathrm{TiO}_{1}$, and an index with small dynamic range, Fe5790. No systematic trends are apparent. Quasi-random scatter is clearly evident, though at a level less than the single-measurement error. It is expected, of course, that the newer versions are incrementally more accurate than the old.

The library of spectra and the 63 averaged indices with errors are available at the CDS.

Acknowledgements. The authors gratefully acknowledge the legacy of David Burstein, without whose labors at the telescope these spectra would never have been recorded, and dedicate this paper to his memory. We also thank former Washington State University undergraduate Angela “A.J.” Marino, whose enthusiasm for polynomial fitting ensured the success of this effort.

\section{References}

Burstein, D., Faber, S. M., Gaskell, C. M., \& Krumm, N. 1984, ApJ, 287, 586 Burstein, D., Faber, S. M., \& González, J. J. 1986, AJ, 91, 1130

Buser, R., \& Kurucz, R. L. 1992, A\&A, 264, 557

Cervantes, J. L., \& Vazdekis, A. 2009, MNRAS, 392, 691

Faber, S. M., Friel, E. D., Burstein, D., \& Gaskell, C. M. 1985, ApJS, 57, 711

Falcón-Barroso, J., Sánchez-Blázquez, P., Vazdekis, A., et al. 2011, A\&A, 532, A95

González, J. J. 1993, Ph.D. Thesis, Univ. California, Santa Cruz, USA

Gorgas, J., Faber, S. M., Burstein, D., et al. 1993, ApJS, 86, 153

Heap, S. R., \& Lindler, D. 2010, BAAS, 42, 494

Prugniel, Ph., \& Soubiran, C. 2001, A\&A, 369, 1048

Robinson, L., \& Wampler, E. J. 1972, PASP, 84, 161

Sánchez-Blázquez, P., Peletier, R. F., Jiménez-Vicente, J., et al. 2006, MNRAS, 371,703

Serven, J., Worthey, G., \& Briley, M. M. 2005, ApJ, 627, 754

Trager, S. C., Worthey, G., Faber, S. M., Burstein, D., \& Gonzalez, J. J. 1998 ApJS, 116, 1

Tukey, J. W. 1958, Ann. Math. Stat. 29, 614

Valdes, F., Gupta, R., Rose, J. A., Singh, H. P., \& Bell, D. J. 2004, ApJS, 152, 251

Worthey, G., \& Ottaviani, D. L. 1997, ApJS, 111, 377

Worthey, G., Faber, S. M., González, J. J., \& Burstein, D. 1994, ApJS, 94, 687 
Table 1. Single measurement index uncertainty and index definitions.

\begin{tabular}{|c|c|c|c|c|c|c|}
\hline Index & $\sigma^{a}$ & Units & $\begin{array}{l}\text { Definition } \\
\text { references }\end{array}$ & $\begin{array}{l}\text { Index } \\
\text { passband }^{b}\end{array}$ & $\begin{array}{l}\text { Blue } \\
\text { pseudocontinuum }{ }^{b}\end{array}$ & $\begin{array}{l}\text { Red } \\
\text { pseudocontinuum }{ }^{b}\end{array}$ \\
\hline $\mathrm{CN}_{1}$ & 0.019 & mag & 1 & $4142.125-4177.125$ & $4080.125-4117.625$ & $4244.125-4284.125$ \\
\hline $\mathrm{CN}_{2}$ & 0.021 & mag & 1 & $4142.125-4177.125$ & $4083.875-4096.375$ & $4244.125-4284.125$ \\
\hline $\mathrm{Ca} 4227$ & 0.315 & $\AA$ & 1 & $4222.250-4234.750$ & $4211.000-4219.750$ & $4241.000-4251.000$ \\
\hline G4300 & 0.373 & $\AA ̊$ & 1 & $4281.375-4316.375$ & $4266.375-4282.625$ & $4318.875-4335.125$ \\
\hline $\mathrm{Fe} 4383$ & 0.451 & $\AA ̊$ & 1 & $4369.125-4420.375$ & $4359.125-4370.375$ & $4442.875-4455.375$ \\
\hline Ca4455 & 0.301 & $\AA ̊$ & 1 & $4452.125-4474.625$ & $4445.875-4454.625$ & $4477.125-4492.125$ \\
\hline $\mathrm{Fe} 4531$ & 0.434 & $\AA ̊$ & 1 & $4514.250-4559.250$ & $4504.250-4514.250$ & $4560.500-4579.250$ \\
\hline $\mathrm{C}_{2} 4668$ & 0.674 & $\AA ̊$ & 1 & $4634.000-4720.250$ & $4611.500-4630.250$ & $4742.750-4756.500$ \\
\hline $\mathrm{H} \beta$ & 0.229 & $\AA ̊$ & 1 & $4847.875-4876.625$ & $4827.875-4847.875$ & $4876.625-4891.625$ \\
\hline Fe5015 & 0.485 & $\AA$ & 1 & $4977.750-5054.000$ & $4946.500-4977.750$ & $5054.000-5065.250$ \\
\hline $\mathrm{Mg}_{1}$ & 0.010 & mag & 1 & $5069.125-5134.125$ & $4895.125-4957.625$ & $5301.125-5366.125$ \\
\hline $\mathrm{Mg}_{2}$ & 0.010 & mag & 1 & $5154.125-5196.625$ & $4895.125-4957.625$ & $5301.125-5366.125$ \\
\hline $\operatorname{Mg} b$ & 0.271 & $\AA$ & 1 & $5160.125-5192.625$ & $5142.625-5161.375$ & $5191.375-5206.375$ \\
\hline Fe5270 & 0.286 & $\AA ̊$ & 1 & $5245.650-5285.650$ & $5233.150-5248.150$ & $5285.650-5318.150$ \\
\hline $\mathrm{Fe} 5335$ & 0.274 & $\AA$ & 1 & $5312.125-5352.125$ & $5304.625-5315.875$ & $5353.375-5363.375$ \\
\hline Fe5406 & 0.201 & $\AA ̊$ & 1 & $5387.500-5415.000$ & $5376.250-5387.500$ & $5415.000-5425.000$ \\
\hline Fe5709 & 0.181 & $\AA ̊$ & 1 & $5696.625-5720.375$ & $5672.875-5696.625$ & $5722.875-5736.625$ \\
\hline $\mathrm{Fe} 5782$ & 0.214 & $\AA$ & 1 & $5776.625-5796.625$ & $5765.375-5775.375$ & $5797.875-5811.625$ \\
\hline $\mathrm{Na} \mathrm{D}$ & 0.236 & $\AA$ & 1 & $5876.875-5909.375$ & $5860.625-5875.625$ & $5922.125-5948.125$ \\
\hline $\mathrm{TiO}_{1}$ & 0.010 & $\AA$ & 1 & $5936.625-5994.125$ & $5816.625-5849.125$ & $6038.625-6103.625$ \\
\hline $\mathrm{TiO}_{2}$ & 0.009 & $\AA$ & 1 & $6189.625-6272.125$ & $6066.625-6141.625$ & $6372.625-6415.125$ \\
\hline $\mathrm{H} \delta_{A}$ & 0.779 & $\AA ̊$ & 2 & $4083.500-4122.250$ & $4041.600-4079.750$ & $4128.500-4161.000$ \\
\hline $\mathrm{H} \gamma_{A}$ & 0.555 & $\AA ̊$ & 2 & $4319.750-4363.500$ & $4283.500-4319.750$ & $4367.250-4419.750$ \\
\hline $\mathrm{H} \delta_{F}$ & 0.451 & $\AA$ & 2 & $4091.000-4112.250$ & $4057.250-4088.500$ & $4114.750-4137.250$ \\
\hline $\mathrm{H} \gamma_{F}$ & 0.298 & $\AA$ & 2 & $4331.250-4352.250$ & $4283.500-4319.750$ & $4354.750-4384.750$ \\
\hline $\mathrm{CO} 4685$ & 0.822 & $\AA ̊$ & 3 & $4626.400-4743.300$ & $4557.300-4589.500$ & $4805.100-4835.300$ \\
\hline CO5161 & 0.169 & $\AA$ & 3 & $5154.300-5167.300$ & $5108.400-5138.800$ & $5188.400-5202.000$ \\
\hline $\mathrm{CNO} 4175$ & 0.958 & $\AA ̊$ & 3 & $4129.400-4219.800$ & $4082.500-4123.300$ & $4243.300-4284.200$ \\
\hline $\operatorname{Mg} 4780$ & 0.302 & $\AA ̊$ & 3 & $4760.800-4798.800$ & $4738.900-4757.300$ & $4819.800-4835.500$ \\
\hline Si4101 & 0.316 & $\AA$ & 3 & $4093.300-4107.800$ & $4057.900-4075.300$ & $4111.500-4131.000$ \\
\hline $\mathrm{Si} 4513$ & 0.364 & $\AA$ & 3 & $4490.800-4534.600$ & $4448.700-4487.600$ & $4541.100-4572.700$ \\
\hline S4693 & 0.261 & $\AA ̊$ & 3 & $4680.600-4704.400$ & $4652.200-4677.300$ & $4717.600-4737.700$ \\
\hline K4042 & 0.285 & $\AA ̊$ & 3 & $4035.900-4047.500$ & $4014.900-4029.000$ & $4054.700-4070.400$ \\
\hline $\mathrm{Sc} 4312$ & 0.381 & $\AA$ & 3 & $4303.000-4320.700$ & $4286.100-4300.600$ & $4335.800-4354.400$ \\
\hline Sc6292 & 0.341 & $\AA ̊$ & 3 & $6265.100-6318.800$ & $6199.200-6231.100$ & $6320.900-6366.500$ \\
\hline Ti4296 & 0.334 & $\AA ̊$ & 3 & $4283.600-4308.200$ & $4253.800-4274.400$ & $4324.500-4343.000$ \\
\hline Ti4533 & 0.180 & $\AA$ & 3 & $4525.300-4541.100$ & $4486.300-4506.200$ & $4548.700-4561.300$ \\
\hline Ti5000 & 0.352 & $\AA$ & 3 & $4973.100-5026.100$ & $4930.600-4959.400$ & $5035.400-5052.500$ \\
\hline V4112 & 1.193 & $\AA ̊$ & 3 & $4086.400-4138.500$ & $4057.100-4074.300$ & $4145.000-4160.500$ \\
\hline V4928 & 0.180 & $\AA$ & 3 & $4919.600-4937.400$ & $4899.800-4914.800$ & $4951.800-4965.200$ \\
\hline $\mathrm{Cr} 4264$ & 0.532 & $\AA ̊$ & 3 & $4246.800-4281.300$ & $4179.300-4195.600$ & $4310.700-4334.700$ \\
\hline Cr5206 & 0.149 & $\AA$ & 3 & $5198.900-5213.600$ & $5130.000-5149.200$ & $5240.300-5257.000$ \\
\hline Mn4018 & 3.188 & $\AA$ & 3 & $3992.600-4044.400$ & $3940.900-3962.300$ & $4096.300-4121.500$ \\
\hline Mn4061 & 0.372 & $\AA$ & 3 & $4443.300-4477.700$ & $4386.800-4406.900$ & $4506.100-4530.300$ \\
\hline Mn4757 & 0.252 & $\AA$ & 3 & $4741.500-4773.400$ & $4715.600-4737.800$ & $4792.400-4812.100$ \\
\hline Fe4058 & 0.902 & $\AA$ & 3 & $4037.900-4078.400$ & $4009.000-4031.100$ & $4107.600-4125.700$ \\
\hline $\mathrm{Fe} 4930$ & 0.273 & $\AA ̊$ & 3 & $4907.000-4943.800$ & $4894.500-4907.000$ & $4943.800-4954.500$ \\
\hline $\mathrm{Ni4292}$ & 0.392 & $\AA ̊$ & 3 & $4279.600-4305.400$ & $4260.900-4275.300$ & $4307.400-4323.600$ \\
\hline Ni4910 & 0.235 & $\AA ̊$ & 3 & 4897.400-4922.100 & $4879.300-4895.800$ & $4953.200-4969.400$ \\
\hline Ni4976 & 0.227 & $\AA$ & 3 & $4966.000-4986.300$ & $4951.500-4964.400$ & $5016.800-5028.900$ \\
\hline Ni5592 & 0.210 & $\AA$ & 3 & $5578.300-5605.400$ & $5521.100-5542.700$ & $5612.600-5632.600$ \\
\hline $\mathrm{Cu} 5217$ & 0.218 & $\AA ̊$ & 3 & $5209.100-5225.300$ & $5160.900-5186.900$ & $5247.500-5275.800$ \\
\hline Cu5780 & 0.149 & $\AA ̊$ & 3 & $5772.300-5787.300$ & $5720.100-5753.200$ & $5808.000-5830.800$ \\
\hline Zn4720 & 0.207 & $\AA ̊$ & 3 & $4708.700-4731.500$ & $4652.700-4670.000$ & $4755.400-4775.500$ \\
\hline Ba4552 & 0.169 & $\AA ̊$ & 3 & $4545.400-4558.500$ & $4522.200-4535.000$ & $4559.500-4578.800$ \\
\hline Ba4933 & 0.190 & $\AA$ & 3 & $4922.800-4943.300$ & $4902.700-4916.100$ & $4967.200-4992.700$ \\
\hline Ba6142 & 0.192 & $\AA$ & 3 & $6130.700-6152.600$ & $6105.800-6128.200$ & $6176.300-6209.600$ \\
\hline Sr4076 & 0.424 & $\AA$ & 3 & $4069.000-4082.200$ & $4038.400-4055.600$ & $4105.300-4126.800$ \\
\hline Eu4592 & 0.165 & $\AA$ & 3 & $4584.800-4598.800$ & $4533.500-4550.800$ & $4626.300-4645.700$ \\
\hline $\mathrm{H} \beta$ emiss & 0.154 & $\AA ̊$ & 4 & $4851.320-4871.320$ & $4815.000-4845.000$ & $4880.000-4930.000$ \\
\hline O3emiss1 & 0.294 & $\AA$ & 4 & $4948.920-4987.920$ & $4885.000-4935.000$ & $5030.000-5070.000$ \\
\hline O3emiss 2 & 0.160 & $\AA$ & 4 & $4996.850-5016.850$ & $4885.000-4935.000$ & $5030.000-5070.000$ \\
\hline $\mathrm{H} \beta_{0}$ & 0.261 & $\AA ̊$ & 5 & $4839.275-4877.097$ & $4821.175-4838.404$ & $4897.445-4915.845$ \\
\hline
\end{tabular}

Notes. ${ }^{(a)}$ Expected single-measurement uncertainty for most stars in the library based on the line strength standard star observations. ${ }^{(b)}$ The wavelength spans for pseudocontinua and index passbands are given in units of $\AA$.

References. (1) Trager et al. (1998); (2) Worthey \& Ottaviani (1997); (3) Serven et al. (2005); (4) González (1993); (5) Cervantes \& Vazdekis (2009). 